\title{
Intelligent Healthcare Platform Solution and Medication Management System for Elderly People with Long Term Health Conditions
}

\author{
Riyad ELSAADI ${ }^{1}$, Mahmoud SHAFIK \\ College of Science and Engineering, University of Derby, Derby, \\ DE22 $3 A W, U K$
}

\begin{abstract}
Healthcare and NHS faces many challenges in monitoring health conditions specially for patients with long term health conditions and the elderly. The forward view for healthcare providers and the NHS is moving from the hospital routine medical checks towards home environment care with the use of smart IoT and AI. Medication errors and missed medication across the globe, is the main source of harm to the public health. Technologies, specifically wireless health technologies are potential solutions for medication error and medication nonadherence in tracking patients' medication. This paper presented a solution by developing a real-time wireless sensor network to monitor and check patient's health condition using devices that transmits data from homes wirelessly to the relevant (caregiver, GP, Hospitals and specialist doctors). The proposed system benefits form the use of algorithms, which is used to provide the quality and quick health care advice to the patients at home. The algorithm processes the data from the database webserver. The database stores the patient health history conditions with all measurement obtained from the devices, such as blood pressure, blood glucose, heart rate and body temperature. This data is processed in machine learning algorithm to generate notifications for any changes occur in user's health and by checking their history records. ML can detect patterns within patient healthcare records and inform clinicians of any anomalies.
\end{abstract}

Keywords. Adhoc, Assisted Living, Healthcare AI, Remote Healthcare Monitoring, Python, Webserver, Android Apps, Database.

\section{Introduction}

Our demography faces a period of economic and social changes, one of most challenging affects is the increase in the population per average age across the whole world. People of ages 60 years and older will proceed the children in the coming years by 2047. This would result in the hospitals and care homes will face difficulties in supporting this great demand, which drives the local councils and the government to help the private and the public health care sectors. The need to establish systems for people must be healthy and live more freely, by investing in more preventative care institutions and researcher need to link this gap. The solution is to link the cities with modern technology as known smart cities and people's health must start from their homes. The health care monitoring system specially for the elderly need prioritising. So, that they can live independently and

${ }^{1}$ Corresponding Author. r.elsaadi@derby.ac.uk 
healthy, keeping track of their health by the professionals in the field connected all at once from their comfort homes to the GP.

A revolution in home healthcare has the potential to occur because of the pervasiveness of computing today. This could be a means of solving the economic and social issues caused by population aging. Technological networks could also lend support to informal carers and allow healthcare providers to respond quickly in the event of a crisis [1]. (Dishongh and McGrath, 2010). Due to current social structures, families may not always be geographically proximate, but advances in technology will enable distant family members to play supportive roles without the need for constant travel.

Systems for healthcare in the home will rely upon devices which warn patients of potential illness, enabling them to arrange early intervention [2]. Because wearable sensors and environmental sensors can autonomously process and communicate, these can potentially work in tandem with health providers by acting as the collectors, processors and transmitters of relevant data. Responsive alerts or information could also be provided to the patient via their mobile or computer. Meanwhile, data storage in a facility such as a cloud server or a home computer could offer a long-term perspective on health changes, and even enable better compilation of statistics about health in the wider population.

Systems for health monitoring and provision could make great use of wireless sensor networks and the hardware that accompanies them. One key area would be monitoring physiological signals remotely. Other advantages of these networks include their capacity to monitor in real time, self-organisation, reconfiguration, portability and ease of deployment. Healthcare's quality, efficiency and safety could be considerably improved by IT, and surveys do suggest that greater investment in this area is planned. One of the most significant areas for potential improvement is the quality of healthcare service. However, there are difficulties, such as that implementing IT is both costly and complex and may require investment in workers both external and internal, where culture change may be needed. There have been multiple efforts in both public and private sector healthcare to drive implementation of improved IT systems. In future, increased standardisation, a drive towards interoperability and monetary incentives could be utilised to add further motivation [3].

\section{Proposed Ad-hoc System Architecture}

Figure 1 illustrates the proposed architecture of the integrated ALT system. There are two main components of this architecture; the front-end part is used to incorporate different devices produced by different manufacturers in the ad-hoc base protocol. The front-end will work on different bases of ad-hoc devices protocol and will be able to obtain measurements and readings from the devices. The data is wirelessly transferred to a unique platform incorporating intelligent algorithms and database for processing. Algorithms processing this type of data are primarily present in the second fragment at the back end. To a certain level, the data will be processed and will help in alerting, reporting, and sharing initial findings to family members, doctors, or caregivers. 


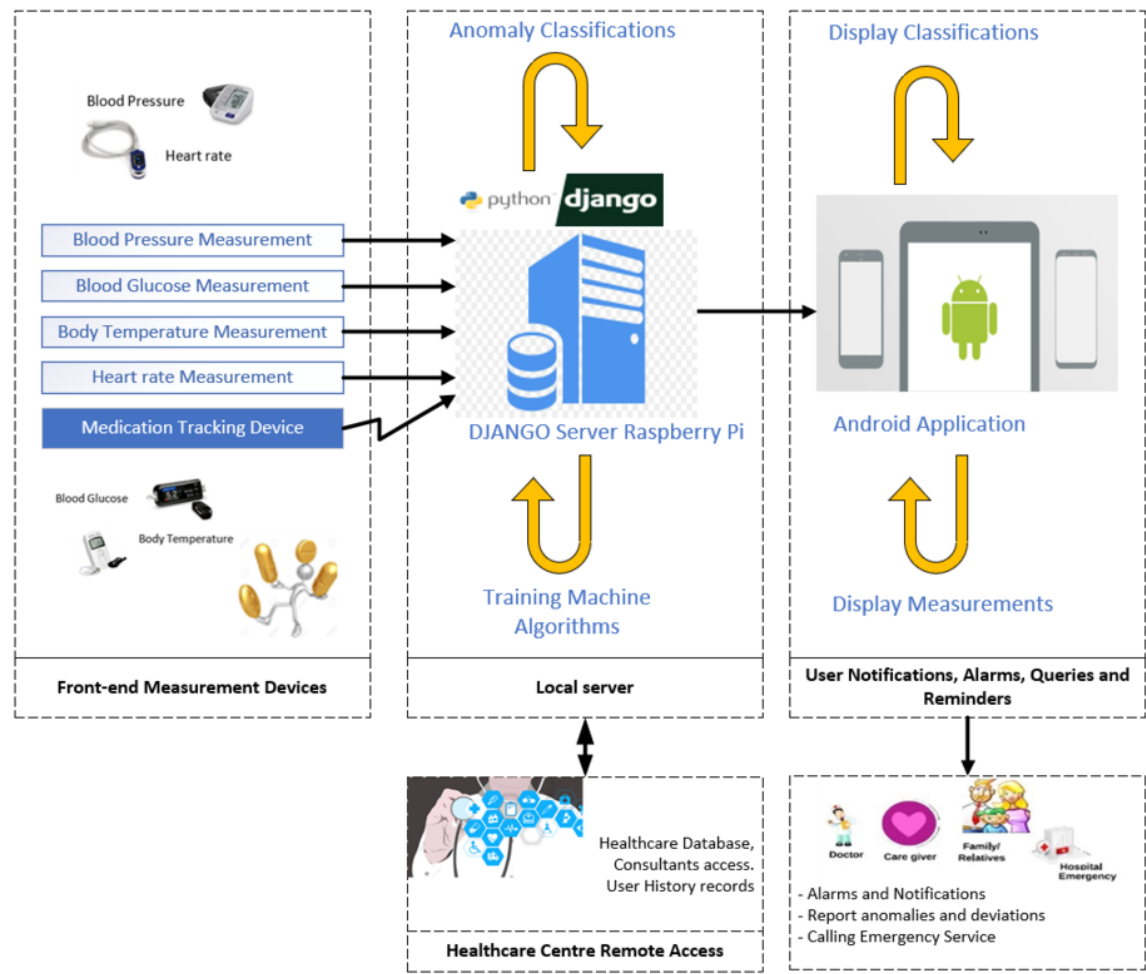

Figure 1 Proposed Solution System Architecture, from the front-end measurement devices and back-end, processing stages and other relevant ad-hoc technology platform

\section{Proposed System Design and Development}

Main module script is the brain of the software as shown below. The script is executed on the Raspberry pi, which uses ARM (Advanced RISC Machine) processor. Raspberry pi acts as host to the different devices. The script calls all the appropriate functions according to the device plugged in.

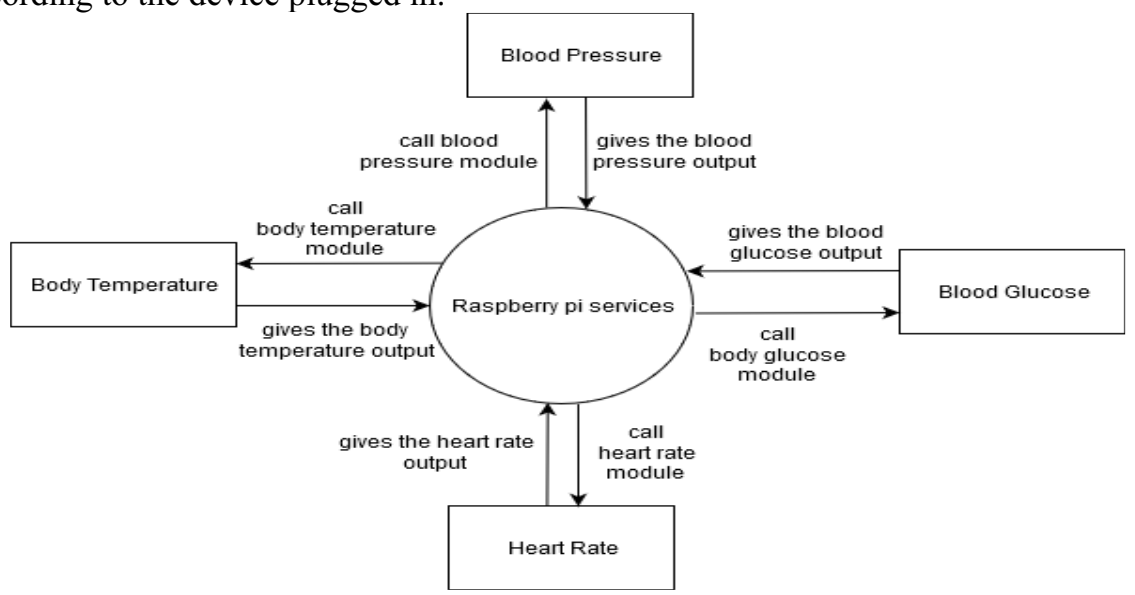

Figure 2 Context diagram of the system design execution. 


\subsection{Blood Pressure Module:}

The module uses the QT based C++ program for hosting the device. Main dependencies including HIDAPI, is a multi-platform library, which allows an application to interface with USB and Bluetooth HID-Class devices. The API provides the most used HID functions including sending and receiving of input and output to obtain the data and be able to save it to csv format for processing.
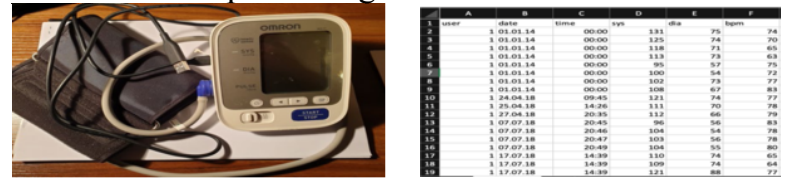

Figure 3: Blood Pressure Device Omron M30 IT with data extracted to csv file.

\subsection{Heart Rate CMS50dPlus}

The device configured by sending commands using Lib USB library in order to get the live data through its serial communication. As soon as it starts getting the data, the program processes it by getting the desired values and saves it to csv file
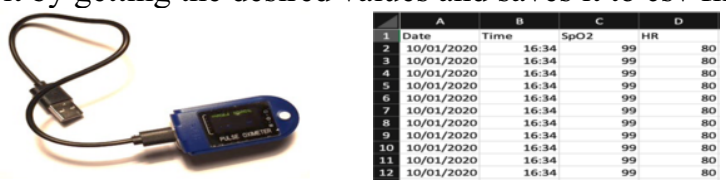

Figure 4: Heart rate device CMS50dPlus

\subsection{Blood Glucose Device}

The device benefits from smart function to store real-time data with according to certain parameter as shown in figure 4 . These are extracted and processed in main module of the system to make decisions and understand the data rates.

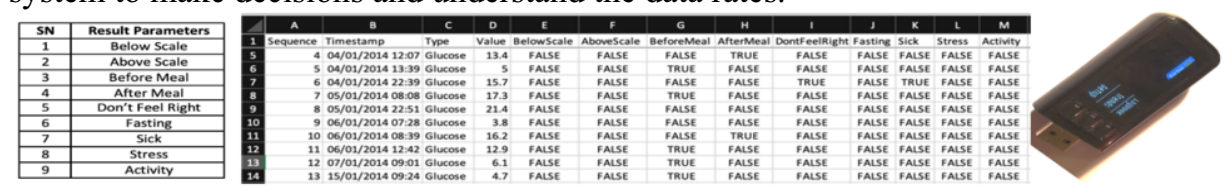

Figure 5: Blood Glucose Device Contour Next USB

\subsection{Body Temperature}

The script written for the body temperature configures the device with intervals, it continuously receives the data until the stopping condition is satisfied. The script will initialise the device and start by "read" get raw data and save the collected data to csv file with its date and time. 


\subsection{Medication Management System}

The Main components the load cell and motion sensor connected with an amplifier to Arduino microcontroller for processing. XBee wireless module is configured so that keeps sending the current weight, and once motion is detected it will send data along with "Detected" on its serial to differentiate between the values and back-end system can then process these data and determine if the medicine was taken or not.

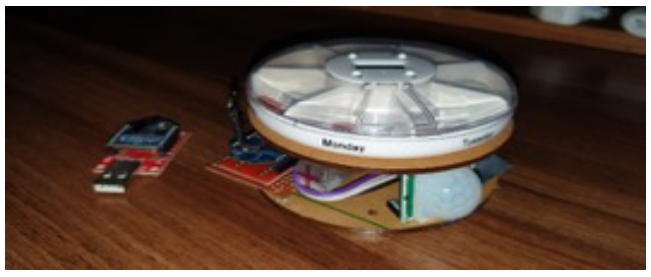

Figure 6: Medication management system design

\section{System Validation, Tests and Results}

Testing the performance of the proposed system is considered with the use of MIMICIII a clinical database to have more sets of data as live example. The validation is done through obtaining the data measurement of the vital health signs. To use any approach of data selection the processing is required in order to perform any data testing and or data testing on the machine learning algorithms. While tabulating the data, there some factors to take in consideration. Such as size of data, and the missing data in malfunctioning system failure. That's why the proposed system has the capability to refetch the data and keep it safe archived from the USB device to the raspberry pi. The system can recover once connection is established with the main server and start sending/ force recheck process on the data using its timestamp.

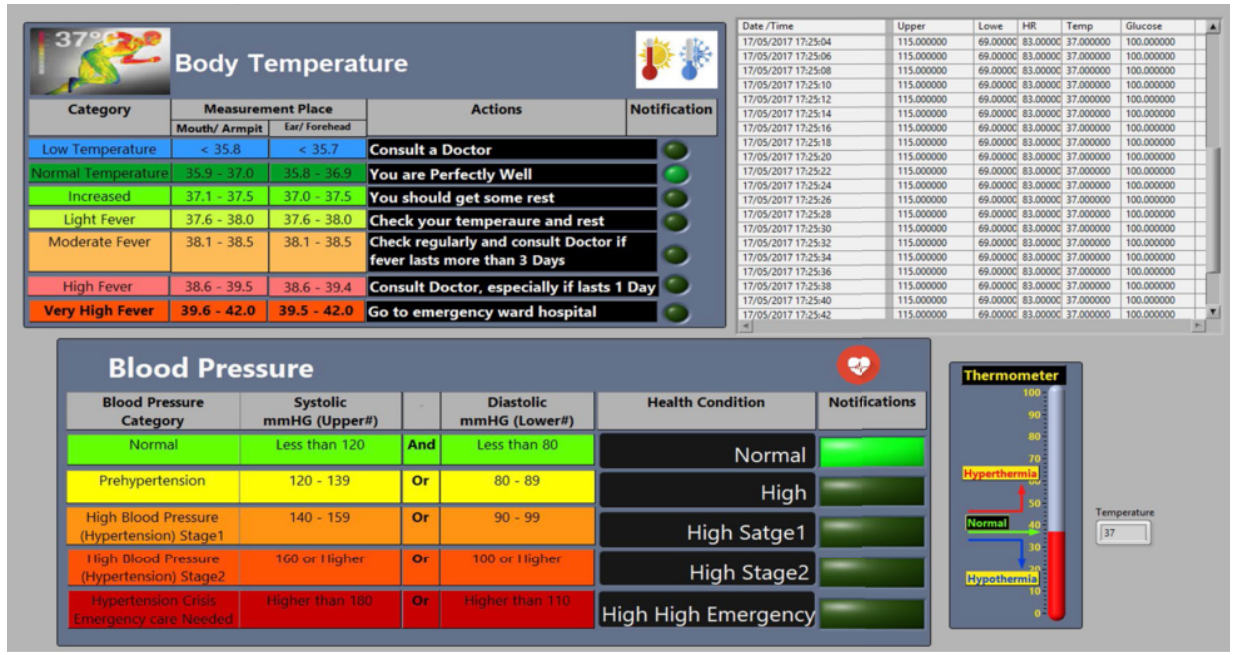

Figure 7: System User Interface and results obtained 


\section{Conclusion}

Presented in this paper an intelligent medical health care solution for elderly people with long term health conditions, to monitor the health conditions and detect the pattern in vital signs anomalies. The proposed developed system enables the continues monitoring and learning the behaviours of the data as they been received from the front-end devices, it will recognise any sudden changes and provide the right intervention. Detecting the patterns for abnormal vital health signs behaviours for the elderly patients is the main key to improve the health monitoring case systems so, they can live independently. The solution in the thesis motivation was based on the development on health care monitoring systems with the elements of interconnection between the different external services and the present health monitoring, taking it into a new level of system integration. This will reduce time, effort as well as costs by eliminating redundant tasks and increase the benefits to the end-users through the applications development process.

\section{Reference:}

[1] Dishongh TJ, McGrath M. Wireless sensor networks for healthcare applications. Artech House; 2010.

[2] McCullagh, Paul J., and Juan Carlos Augusto. "The internet of Things: The potential to facilitate health and wellness." Cepis Upgrade 12, no. 1 (2011): 59-68.

[3] Varghese T, Varghese T, Bhatele M. Remote Blood Pressure Monitoring Using a Wireless Sensor Network. InProceedings of All India Seminar on Biomedical Engineering 2012 (AISOBE 2012) 2013 (pp. 153158). Springer, India.

[4] Mahmoud Shafik \& Alex Robert Campbell (2012), ‘6D Smart Medication Container', Ref. No.: RRS/26769GB, Swindell \& Pearson Ltd.

[5] Mahmoud Shafik (2012), 012), fik (2012), 12), '6D Smart Medication Container', Ref. No.: RRS/26769GB, Swindell \& Pearso59GB, Swindell \& Pearson Ltd.

[6] Riyad Elsaadi \& M Shafik (, 2015), 'An Intelligent Medical Care Solution for Elderly People with Long Term Health Condition Based on Wireless Sensors Network Technology', International Journal of 